\title{
A Study on Freeform Optimization using BIM Technology
}

\author{
Tae Il CHOI, Ho Jun NA, Jin Woo KIM* \\ Hyundai Engineering \& Construction \\ *e-mail:jinwoo@hdec.co.kr
}

\begin{abstract}
Freeform buildings are now designed and constructed globally because of digital design trend, advance construction technology, and landmark value. However, due to the lack of freeform building engineering and construction management experience, it has become a high risk project for construction companies. During construction stage; many design changes, fabrication defects and construction quality issues are being raise, which results project cost increase and schedule delays. This study proposes freeform optimization process to mitigate the risk from happening at construction stage. Using BIM, visualization, parametric model and automation functions, various design alternatives are made to apply curvature analysis, panelization, panelization evaluation, and panel optimization for most efficient solution.
\end{abstract}

\section{KEYWORDS}

Freeform Architecture, Panelization, Panel Optimization

\section{INTRODUCTION}

The rapid development of digital technology has affected the construction environment, especially in the design process, the transition from existing two-dimensional to three-dimensional modeling method, provided architects with environment to express their design philosophy more aggressively.
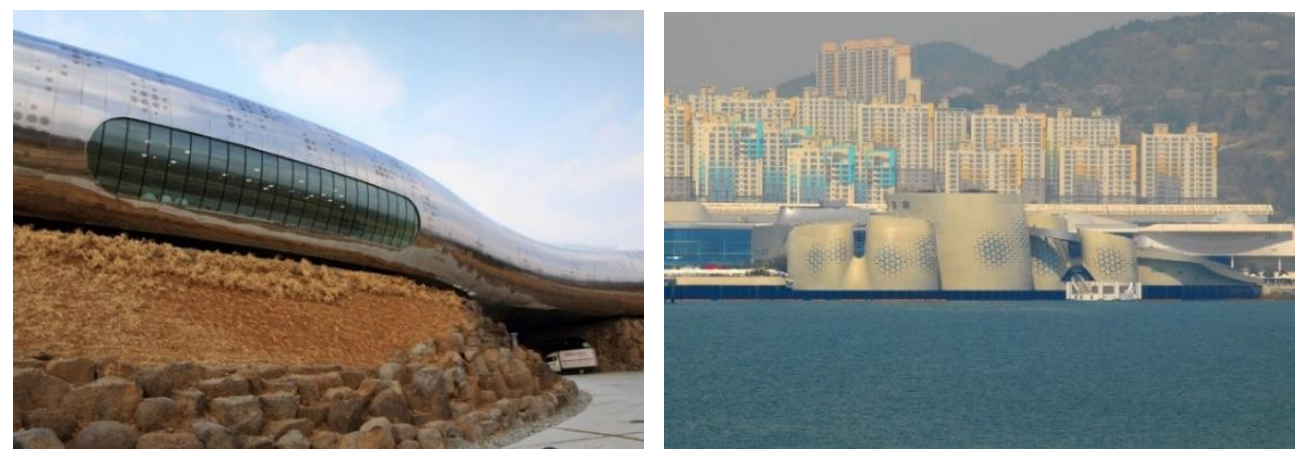

Figure 1. Jeongok Prehistoric Museum (Left) and Yeosu EXPO Theme Pavilion (Right)

Because of its landmark and symbolic presence, the demand on freeform designs are increasing and it's now a major design trend. Projects including Walt Disney Concert Hall, Mercedes-Benz Museum, and BMW Welt and in Korea Dongdaemun Design Plaza, Yeosu EXPO theme pavilion, and Jeongok prehistoric museum are few of many projects.

However, from construction point of view, the lack of engineering method and construction experience led to major delays in construction schedule and increase in cost. In order to 
successfully complete the project, 'pre-engineering', head first involvement in design and engineering phase of project are needed. Especially, in freeform projects, exterior surface consist of numerous curvature with material including concrete, metal, glass, wood and etc. are critical engineering factor which directly related to cost, schedule and efficiency of fabrication and construction.

This study introduces the optimization method on freeform exterior surface, focusing on increasing the efficiency of fabrication and construction but maintaining architect's design intention using BIM technology.

\section{FREEFORM OPTIMIZATION}

\section{Freeform Optimization Process on Exterior Surface}

Freeform optimization process on exterior surface are composed of three major steps: first step is design analysis, second step is panelization and third step is optimization. These three steps were implemented at the design stage of ongoing project in Colombo, Sri Lanka using design documents and 3D models provided by architects and client. The process was carried out using BIM software called Digital Project V1, R5 by Gehry Technologies.

Table 1. Project General Information

\begin{tabular}{cc}
\hline Project Name & Keells City Project \\
Location & Colombo, Sri Lanka \\
Client & John Keells Holdings \\
Architect & Balmond Studio (UK) \\
Construction Started & $2014.03 \sim 2018.03(48 \mathrm{month})$ \\
Total Area & $391,897 \mathrm{~m}^{2}$ \\
Freeform & Retail Roof (Area 37,607 $\left.\mathrm{m}^{2}\right)$ \\
\hline
\end{tabular}
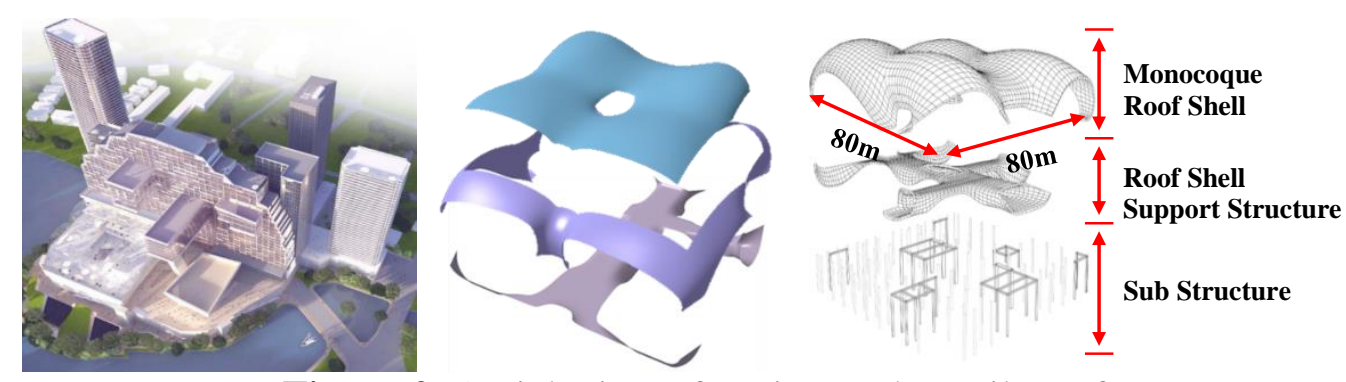

Figure 2. Aerial View of Project and Retail Roof

\section{Freeform Design Analysis}

Design analysis step, preliminary analysis, mainly focuses on gaussian curvature analysis and porcupine curvature analysis applied on surface of geometry. In figure 3, porcupine curvature analysis analyzes the curvature of curves composing the surface and its point of inflection and gaussian curvature analysis analyzes the product of the principal curvatures by representing concave in red and convex in blue. 

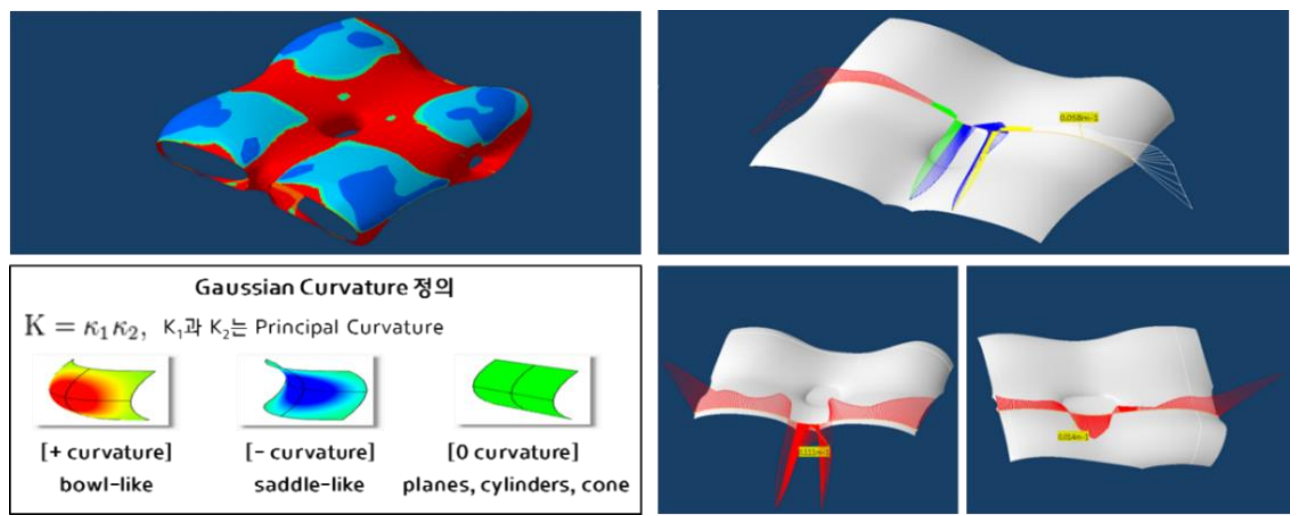

Figure 3. Gaussian (left) and Porcupine (right) Curvature Analysis

The panels can be classified according to the curvature, the flat panel refers to a panel without the occurrence direction of curve, the one-way panel refers to a panel with one direction of curve, and two/or more-way panel refers to a panel with two or more direction of curve.
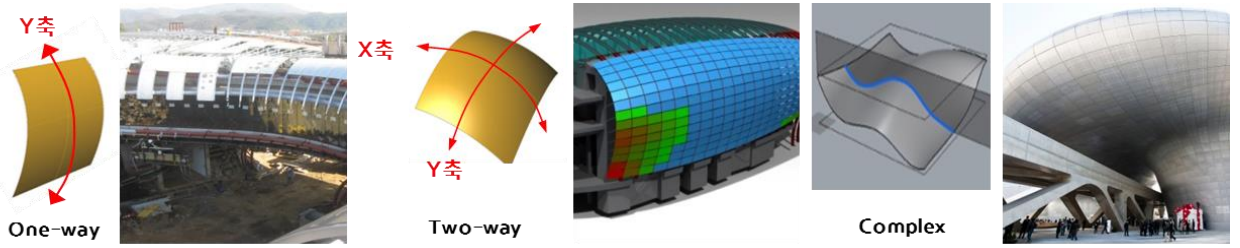

Figure 4. Type of Panel

Result of implementing design analysis step to the project was seventy-nine percent of total surface area was two-way panel and rest, twenty-one percent was flat or one-way panel. In further study, limited the area to top surface of retail roof which is forty-five percent of total surface area.

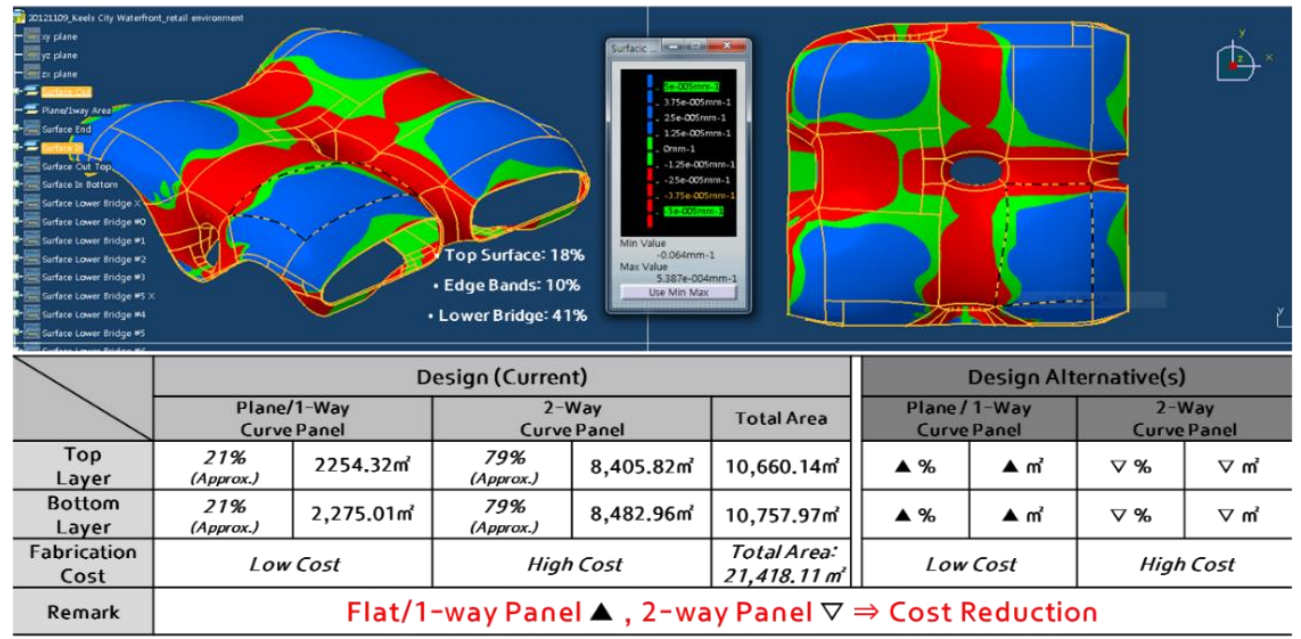

Figure 5. Classification of Panel on Project

\section{Freeform Panelization}

Freeform panelization step is composed of developing and analyzing the developed pattern. 


\section{Pattern Development}

In order to analyze the surface more specifically, patterns are needed to be developed on the surface. This study intersection curve pattern and isoparametric curves pattern are generated. The process was carried out using workbench called Product Knowledge Template, in Digital Project.

The intersection curve pattern is generated by projecting two profile from XY plane to the surface. Two profiles are parametrically controlled to make various range of distance and angle to make alternative pattern. Figure 6 is script in template generating intersection curve pattern on surface and the result of generated intersection curve pattern using template on top surface of retail.

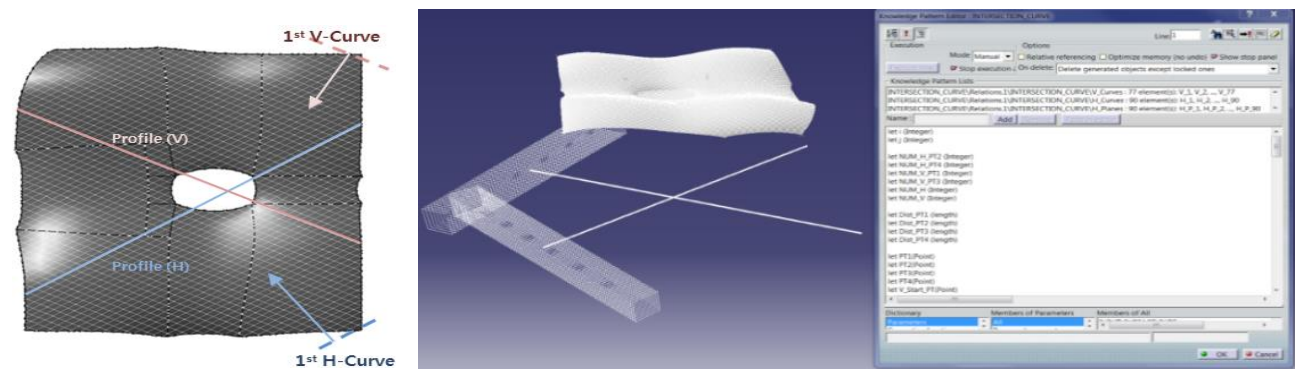

Figure 6. Generated Intersection Curve Pattern and Script in Template

The isoparametric curves pattern is lines running along the surface in the $\mathrm{U}$ and $\mathrm{V}$ directions, representing shape of the surface. This pattern are also parametrically controlled to make various range of distance between curves to make pattern. Figure 7 is script in template generating isoparametric curve pattern on surface and the result of generated isoparametric curve pattern using template on top surface of retail. And using developed pattern, each panel is created with given a unique name.
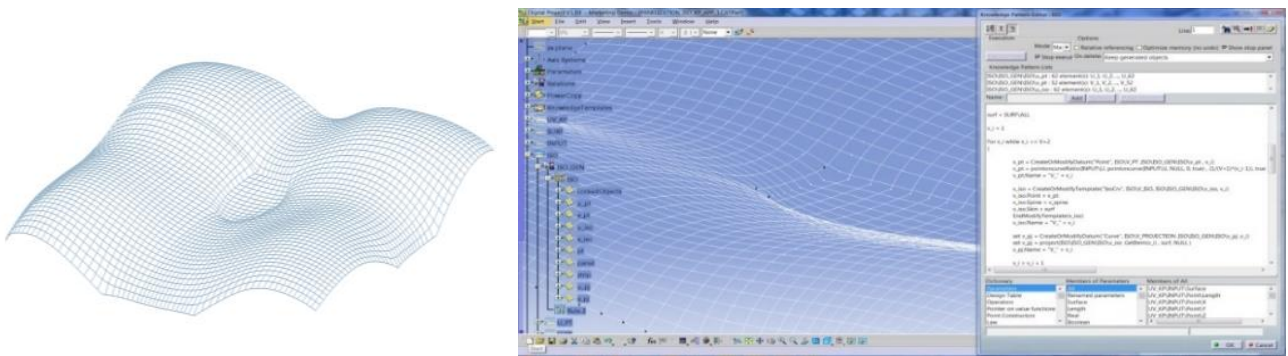

Figure 7. Generated Isoparametric Curve Pattern and Script in Template

\section{Panel Analysis}

After panels were generated with alternative patterns, panels are analyzed to find the best pattern to fabricate and to construct. In order to analyze, all classifies data from panel are extracted in excel such data includes each panel's edges length, surface area, diagonal length between vertices and distance between plane created by three vertices and remainder vertex etc.

\begin{tabular}{|c|c|c|c|c|c|c|c|c|c|c|c|}
\hline & Panel_Name & $\begin{array}{l}\text { Area } \\
\mathrm{cm} 2\end{array}$ & $\begin{array}{l}\text { ED12 } \\
\mathrm{mm}\end{array}$ & $\begin{array}{l}\text { ED13 } \\
\mathrm{mm}\end{array}$ & $\begin{array}{l}\text { ED34 } \\
\mathrm{mm}\end{array}$ & $\begin{array}{l}\text { ED42 } \\
\mathrm{mm}\end{array}$ & ED_MAX & $\begin{array}{l}\text { PE_MAX } \\
\mathrm{mm}\end{array}$ & $\begin{array}{l}\text { MaxLength } \\
\mathrm{mm}\end{array}$ & $\begin{array}{l}\text { MaxHeight } \\
\mathrm{mm}\end{array}$ & $\mathrm{F}$ \\
\hline 1 & PAN_1_1 & 8811.713 & 853.254 & 1027.689 & 863.086 & 1028.3 & 1028.3 & 1028.279 & 1372.906 & 9.274 & TRUE \\
\hline 2 & PAN_1_2 & 7965.178 & 863.086 & 920.373 & 869.933 & 920.414 & 920.414 & 920.398 & 1296.998 & 8.558 & TRUE \\
\hline 3 & PAN_1_3 & 7371.232 & 869.933 & 846.21 & 874.763 & 845.939 & 874.763 & 874.667 & 1246.623 & 8.106 & TRUE \\
\hline
\end{tabular}

Figure 8. Extracted Data from Panel 
The result from comparing extracted data between two patterns were isoparametric curve pattern panel's edge length and diagonal length between vertices were more uniform shape than intersection curve pattern and panel shape also matched the architect's design intent. Figure 9 shows the analyzed data using intersection curve pattern and isoparametric curve pattern.
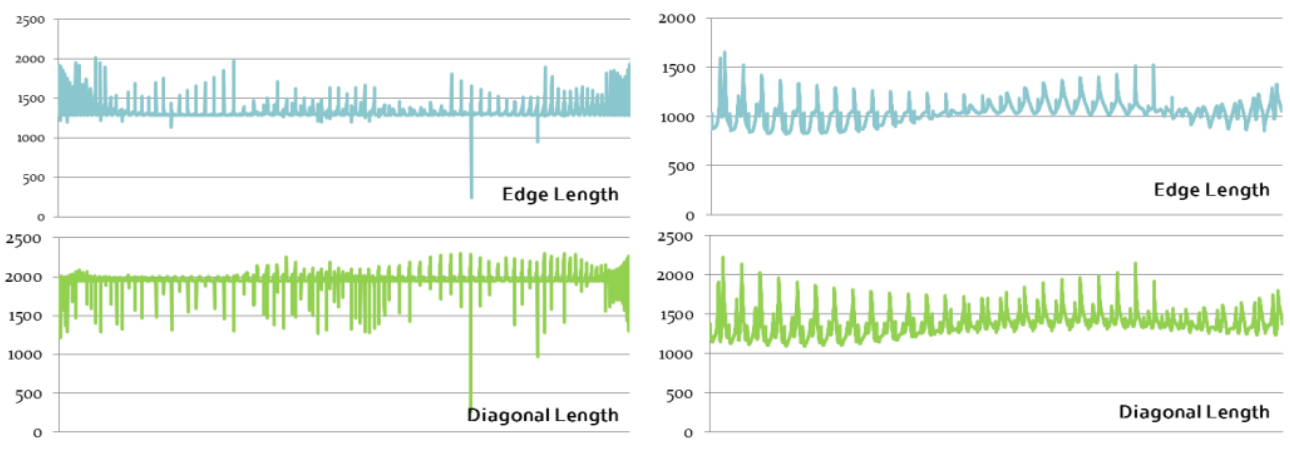

Figure 9. Analyzed Data from Intersection (left) and Isoparametric (right) Curve Pattern

\section{Freeform Optimization}

Optimization Considering Fabrication and Construction Methods. Using panels generated by isoparametric curve pattern, in optimization step, two additional aspects are considered to optimize the panels. First aspect is fabrication method considering fabrication machine and its capability. In order to produce panels, two-way panel are produced using multi point stretching forming(MPSF) machine or cold/heat forming(using profiles) and one-way panel are produced using conventional three point rollers and flat panel are produced using CNC cutting machine. When comparing the fabrication time and cost between these fabrication methods, two-way panel's fabrication cost is as ten times as more expensive comparing to one-way or flat panel. .
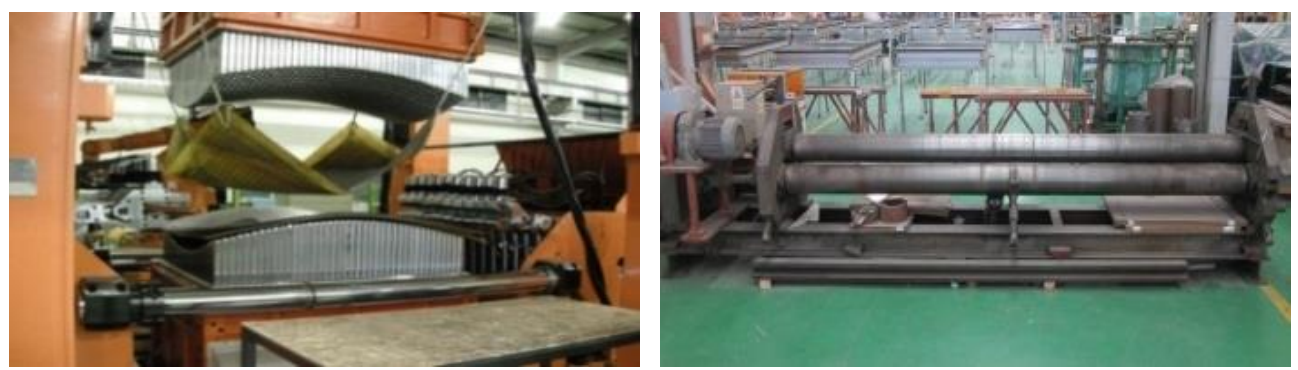

Figure 10. Panel Fabrication Machine: MPSF (left) and Roller (right)

Second aspect is using tolerance and kink angle within the panel; tolerance is given distance off from the original surface and kink angle is angles between neighborhood panels. In case of tolerance and kink angle are given by architect, both parameter are used to increase the fabrication and construction efficiency, larger value meaning more number of alternative generation and greater cost and time efficiency but less following design intention. 

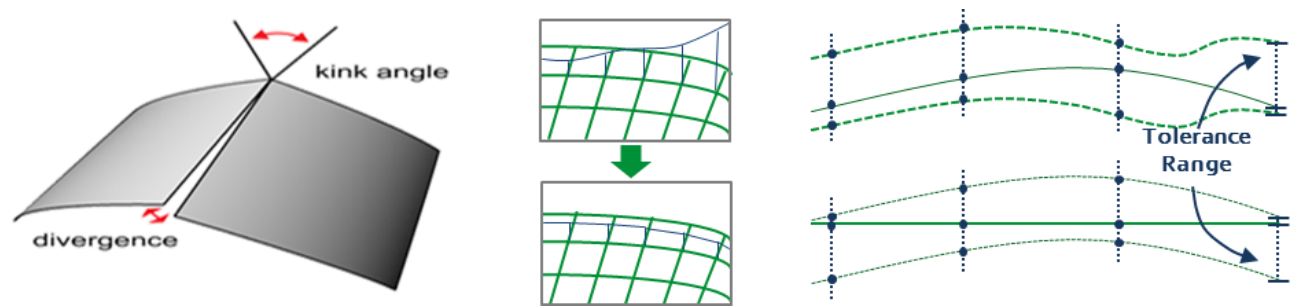

Figure 11. Tolerance and Kink Angle

Optimization and Analysis Application. Tolerance of five millimeters and kink angle of two degrees are applied to panel generated by isoparametric curve pattern. Figure 12 shows the optimization template that applied to each panels, template are generated by using two profile and eight degree of freedom (DOF) and tolerance are measured using seventeen sample points on the panel and kink angles are measured using twelve points on the panel's edges. The optimization template runs total of 600 loops for each panel to generate closest possible alternative in given tolerance and kink angle value.
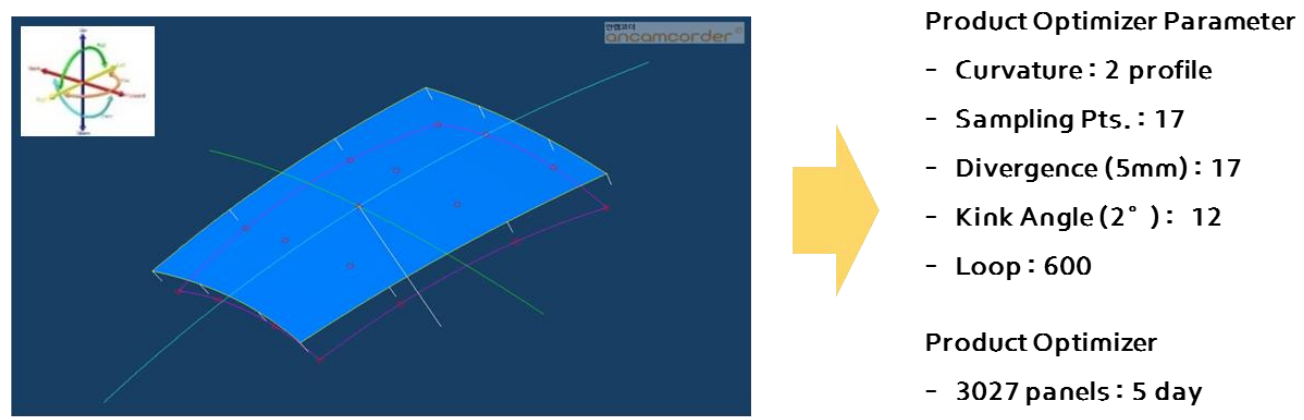

Figure 12. Optimization Template using Profile, DOF, and Sample Point

In this study, the optimization template was applied on 3,027 panels, this process took 5 days. The original panel analysis result was 161 panels (5\%) was flat and 2,866 panels (95\%) was two-way or one-way panel. After optimization process, 888 panels (29\%) was flat, 2,106 panels (70\%) was two-way, and 33 panels (1\%) was one-way. Comparing them both 760 two-way panel was either changed to flat or one-way. Considering fabrication cost difference between two-way and one-way or flat panel are near 300 dollars, total 220 thousand dollars were saved from fabrication cost.
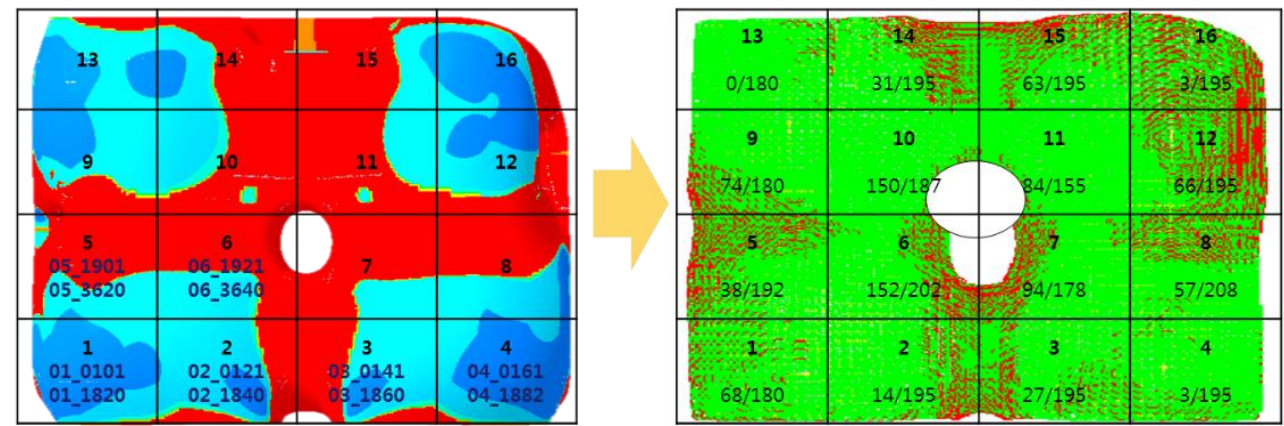

Figure 13. Result from Optimization 
Table 2. Result from Optimization

\begin{tabular}{ccc}
\hline & Panelization & Optimization \\
\hline two-way panel & $2,866(95 \%)$ & $2,106(70 \%)$ \\
one-way panel & $161(5 \%)$ & $33(1 \%)$ \\
flat panel & 3,027 & $888(29 \%)$ \\
total & & 3,027 \\
\hline
\end{tabular}

\section{CONCLUSIONS}

Because of digital design trend, advance construction technology, and landmark value; freeform buildings are now designed and constructed globally. However, due to the lack of freeform building design and construction management experience, it has become a high risk project. During construction stage; many design changes, fabrication defects and construction quality issues are being raise, which results project cost increase and schedule delays.

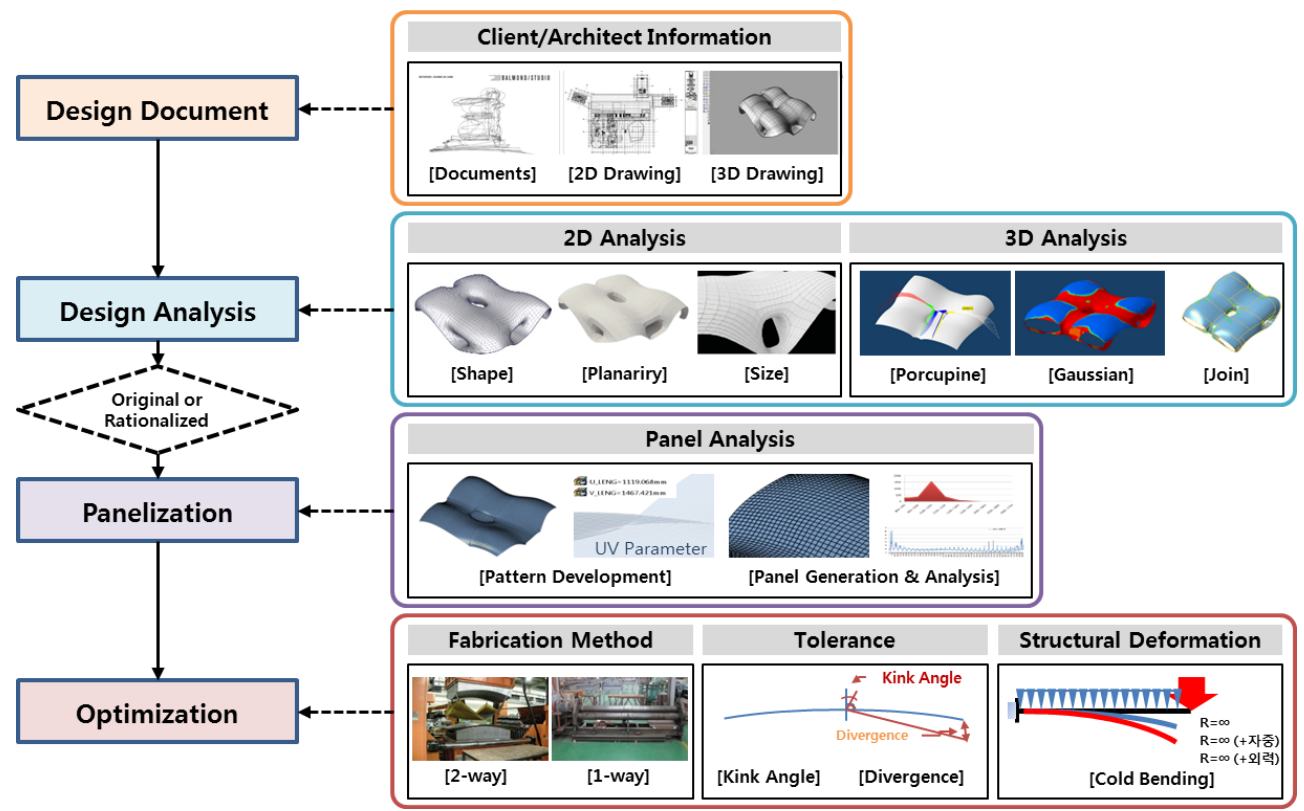

Figure 14. Freeform Optimization Process

Based on this study, we expect to apply freeform optimization process to mitigate the risk from happening at construction stage. Currently, freeform optimization process are applied in tender and engineering/construction stage on ongoing project to verify and upgrade to the field application problems that we might not have considered. And also, we are expanding our study to integrate form finding including structure analysis.

\section{REFERENCES}

Dennis R. Shelden (1997) "Digital Surface Representation and the Constructability of Gehry's Architecture", MIT.

Branko Kolarevic and Kevin Klinger (2008) "Manufacturing Material Effects: Rethinking Design and Making in Architecture", Taylor \& Francis Group, 2008. 
Branko Kolarevic (2003) "Architecture in the Digital Age Design and Manufacturing", Taylor \& Francis Group.

Jeongwon Yoo (2012) "BIM-based Panelizing Optimization for Architectural Freeform Surfaces", Seoul National University.

Seonwoo Kim (2009) "A Study on Classification and Optimization of Free-Form Panel considering Fabrication Methods: DDP”, Yonsei University, 2009.

Jiyeon Ahn (2011) "A Study on the Lessons Learned for Improving Efficiency of Free-Form Facade Construction", Seoultech University.

Soyeon Choi (2012) "A Study on the Free-Form Construction Process based on DDP", Yonsei University.

Soonho Kwon (2011) "A Study on the Problem Analysis and Quality Improvement in Fabricating Free-Form Building's Facade Panels through Mock-up Panels Production”, Korean journal of construction engineering and management, 12(3), pp. 11-21.

Hongsik Park (2009) "A study on a surface generation method of free-form architecture using a planar quadrilateral mesh technique", Hanyang University. 